\title{
Pharmacogenetic guidance: individualized medicine promotes enhanced pain outcomes
}

This article was published in the following Dove Press journal: Journal of Pain Research

\author{
Lisa Lynn Dragic' \\ Erica L Wegrzyn² \\ Michael E Schatman ${ }^{3-5}$ \\ Jeffrey Fudin ${ }^{2,6}$ \\ 'Central Arkansas Veterans Healthcare \\ System, Little Rock, AR, USA; \\ ${ }^{2}$ Department of Pharmacy, Albany \\ Stratton VA Medical Center, Albany, \\ NY, USA; ${ }^{3}$ Research and Network \\ Development, Boston Pain Care, \\ Waltham, MA, USA; ${ }^{4}$ Public Health and \\ Community Medicine, Tufts University \\ School of Medicine, Boston, MA, USA; \\ ${ }^{5}$ Department of Public Health and \\ Community Medicine, Tufts University \\ School of Medicine, Boston, MA, \\ USA; ${ }^{6}$ Scientific and Clinical Affairs, \\ Remitigate, LLC, Delmar, NY, USA
}

Correspondence: Jeffrey Fudin Scientific and Clinical Affairs, Remitigate LLC, 357 Delaware Avenue, \#124,

Delmar, NY 12054, USA

Tel + I $5|8558565|$

Email jeffrey.fudin@remitigate.com

\begin{abstract}
The use of pharmacogenomics has become more prevalent over the past several years in treating many disease states. Several cytochrome P450 enzymes play a role in the metabolism of many pain medications including opioids and antidepressants. Noncytochrome P450 enzymes such as methylenetetrahydrofolate reductase (MTHFR) and catechol- $O$-methyl transferase (COMT) also play a role in the explanation of opioid dosage requirements as well as in response to certain antidepressants. We present the case of a patient with reduced COMT and MTHFR expression treated with leucovorin $10 \mathrm{mg}$ daily for the management of chronic pain. The use of leucovorin in this patient decreased pain scores, which were clinically significant and increased functionality. This case demonstrates the importance of pharmacogenetics testing in patients, as this can help direct providers to better therapeutic options for their patients.
\end{abstract}

Keywords: pharmacogenetic, depression, pain, MTHFR, COMT, methyl tetrahydrofolate reductase, catechol- $O$-methyltransferase

\section{Introduction}

Pharmacogenetics (PGT) plays an important role in several disease states including pain management and mental health disorders. The use of pharmacogenomics has become more prevalent over the past several years in treating various disease states, and over 150 drug manufacturers' inserts include statements on dosing depending on these parameters. Several cytochrome (CYP) P450 enzymes play a role in the metabolism of many pain medications, including opioids and antidepressants. Genetic phenotypes vary among worldwide populations and even within populations. ${ }^{1}$ This phenomenon is known as polymorphism. Aside from CYP450 polymorphisms, there are many important genetic factors that affect response to and side effects of various medications that are commonly used in pain patients. This is especially important in patients with long-term pain when considering that various mental health disorders are common. ${ }^{2}$ Examples of nonCYP450 polymorphisms include methylenetetrahydrofolate reductase (MTHFR) and catechol- $O$ methyl-transferase (COMT), both of which play an important role in opioid and antidepressant response. We present a unique and interesting case of a patient with reduced COMT and MTHFR genes and the impact of initiating leucovorin. Ethical considerations for including PGT in the differential are discussed in terms of cost justification and outcomes. 
Table I

\begin{tabular}{lll}
\hline Examples of medications & Enzyme & Metabolite \\
\hline Citalopram & 2CI9, 3A4, 2D6 & Desmethylcitalopram \\
Venlafaxine & 2D6 & O-desmethylvenlafaxine \\
Bupropion & 2B6 & Hydroxybupropion \\
\hline
\end{tabular}

\section{Background}

PT is a 48-year-old man with a past medical history significant for attention-deficit hyperactivity disorder (ADHD), obstructive sleep apnea, fibromyalgia, posttraumatic stress disorder, and chronic low-back pain. The patient presented in October 2016 with worsening fibromyalgia pain and previous extreme sensitivity to antidepressants as outlined later. Prior to the patient's visit to a comprehensive pain clinic, the patient was started on duloxetine, and after 5 days the patient reported a "debilitating headache and hangover effect." The patient also reported similar side effects to venlafaxine, citalopram, sertraline, bupropion, and mirtazapine.

Because of the patient's reactions to multiple medications, PT was sent for a PGT by saliva swab. PT was tested for 2C19, 2D6, 3A4/5, MTHFR, and COMT, all of which are responsible for the metabolism of many medications. ${ }^{3,4}$ For a summary of results, see Table 1 .

\section{PGT results}

PGT testing yielded the following: $C O M T$ - reduced activity, MTHFR - reduced activity, CYP3A4 and CYP3A5 intermediate metabolizer, CYP2C19 - normal metabolizer CYP2D6 - normal metabolizer, and UGT2B15 - normal metabolizer.

\section{Role of COMT and MTHFR}

The patient exhibited reduced COMT activity, specifically the expression of the VAL158MET genotype. COMT is an enzyme that is responsible for the breakdown of serotonin, dopamine, norepinephrine, and other common neuroamines within neuronal junctions. ${ }^{5-7}$ The VAL158Met allele is associated with low COMT activity, which corresponds to increased concentrations of dopamine and norepinephrine in the cortical region. ${ }^{5-7}$ PT was also receiving methylphenidate, which blocks the reuptake of norepinephrine and dopamine into presynaptic neurons, resulting in increased dopaminergic and noradrenergic activity in the prefrontal cortex. We note that PT also exhibited reduced MTHFR activity, specifically the $677 \mathrm{~T}$ and A1298C genotypes. MTHFR is responsible for converting 5,10-methylenetetrahydrofolate to 5-methyltetrahydrofolate, and 5-methyltetrahydrofolate is the predominant circulating form of folate. ${ }^{8}$ MTHFR C677T polymorphism is associated with a reduction in the bioavailability of folate and folate metabolites. Thus, we expected PT to demonstrate a decreased ability to convert folic acid to its active form, essentially mimicking low dietary folate intake. ${ }^{8}$ Not only have reduced folate levels been linked to depression, but also to attention deficit disorder. ${ }^{8-10}$

At PT's subsequent pain management visit in October 2016, he reiterated his extreme sensitivity to antidepressants and insufficient response to methylphenidate both for depression and ADHD. Theoretically, PT should have exhibited an increased response to methylphenidate due to reduced COMT activity; however, there is a possibility that the patient may have uncontrolled attention deficit disorder despite treatment due to reduced levels of usable folate. MTHFR activity is associated with poor or limited response to antidepressants, pain medications, and methylphenidate. ${ }^{10}$ Therefore, we hypothesized that PT could benefit from the addition of folate supplementation such as L-methylfolate or lecuovorin for pain, depression, and perhaps ADHD. ${ }^{10}$

\section{Folate supplementation}

L-Methylfolate is the active form of folate, and is the only form of folate that crosses the blood-brain barrier. ${ }^{10}$ Studies suggest that the use of L-methylfolate as an adjunctive agent to specific serotonin reuptake inhibitor and serotonin norepinephrine reuptake inhibitor treatment is associated with improved depression treatment outcomes. ${ }^{10}$ Additionally, supplementation of L-methylfolate may provide increased efficacy of methylphenadiate. ${ }^{10}$

Since L-methylfolate was not a formulary item within the institution at which PT was being treated, the patient was started on leucovorin (folinic acid) $10 \mathrm{mg}$ PO each morning for folate supplementation in November 2016. Leucovorin is the active form of folate and does not require MTHFR for activation. Possible side effects of leucovorin include neuropathic pain and zinc depletion; therefore, we provided him with zinc sulfate $220 \mathrm{mg}$ PO every evening.

Prior to initiation of leucovorin, PT reported a pain level of 9 out of 10 on a visual analog scale of $0-10$, with 0 signifying no pain and 10 representing the worst pain possible. Just 1 week following the initiation of leucovorin, the patient reported his pain level to be $2-3$ out of 10 . He reported feeling "much better than before and was sharing his positive results with his friends in the medical field and was very grateful for the genetics testing." Eight months later, he remains stable with regard to his pain, depression, and ADHD. He also reported increased functioning with daily activities. 
Although the clinical implications of this case are quite evident, the ethical issues associated with it are both obvious and subtle. First, and perhaps foremost, PGT should be performed in all cases in which patients demonstrate a combination of both nonresponsiveness and hypersensitivity to numerous medications. Unfortunately, in the United States, PGT is underutilized, with much of this failure to regularly use such technology due to insurers' refusal to cover its costs. This failure has been empirically demonstrated to reduce patients' willingness to utilize PGT, irrespective of their interest in such tests. ${ }^{11}$ Fortunately, the Veterans' Affairs Medical System through which PT was treated has the wisdom to recognize that cost containment is not necessarily synonymous with cost efficiency.

Second, while CYP450 polymorphisms seem to receive considerable attention due to their formidable impact on opioid response, clinicians (and payers) need to recognize that a broad range of potential polymorphisms should not be ignored. In the case of PT, he was determined to be a normal metabolizer of CYP2D6 - the most common CYP P450 polymorphism. ${ }^{12}$ "Simple" PGT testing is not necessarily "thorough" PGT, with this case serving as evidence that numerous potential polymorphisms should be considered when patients are nonresponsive or hypersensitive to multiple therapeutic agents.

Finally, PT's good fortune to be treated by open-minded clinicians should not be underestimated. As a sufferer of long-term pain, many hospitals would not have done their due diligence by providing him with a comprehensive PGT panel. Patients with chronic pain, for myriad unfortunate reasons, are expected to respond positively to whatever pharmacologic intervention is initially provided. Those who do not do so are often stigmatized and provided with pejorative labels such as "drug-seekers." ${ }^{13}$ We posit that although all patients with disease states deserve precision medicine approaches such as that received by PT, the stigmatization and marginalization of patients with the disease of chronic pain and criticism for random morphine equivalent daily dose limits in this unfortunate era of frank opiophobia make consideration of the comprehensive use of PGT even more crucial if their condition is to be treated from an optimally ethical perspective.

\section{Consent}

The patient has provided written informed consent for the case details to be published.

\section{Disclosure}

Dr Schatman would like to disclose that he is a consultant for Depomed. Dr Fudin discloses the following: Astra Zeneca (Speakers Bureau), Daiichi Sankyo (Advisory Board), Egalet (consultant, Advisory Board), Quest Labs (Advisory Board), Remitigate, LLC (Owner). The authors report no other conflicts of interest in this work.

\section{References}

1. Kirsh KL, Ehlenberger E, Huskey A, Strickland J, Egan City K, Passik SD. Exploring rates of abnormal pharmacogenetic findings in a pain practice. J Pain Palliat Care Pharmacother. 2014;28(1):28-32.

2. Velly AM, Mohit S. Epidemiology of pain and relation to psychiatric disorders. Prog Neuropsychopharmacol Biol Psychiatry. Epub 2017 May 15.

3. Kapur BM, Lala PK, Shaw JL. Pharmacogenetics of chronic pain management. Clin Biochem. 2014;47(13-14):1169-1187.

4. Ciraulo DA. Pharmacotherapy of Depression. Humana Press: Totowa, NJ; 2004.

5. Matthews N, Vance A, Cummins TD, et al. The COMT Val158 allele is associated with impaired delayed-match-to-sample performance in ADHD. Behav Brain Funct. 2012;8:25.

6. Cheon KA, Jun JY, Cho DY. Association of the catechol-O-methyltransferase polymorphism with methylphenidate response in a classroom setting in children with attention-deficit hyperactivity disorder. Int Clin Psychopharmacol. 2008;23(5):291-298.

7. Bonvicini C, Faraone SV, Scassellati C. Attention-deficit hyperactivity disorder in adults: a systematic review and meta-analysis of genetic, pharmacogenetic and biochemical studies. Mol Psychiatry. 2016;21(11):1643.

8. Gokcen C, Kocak N, Pekgor A. Methylenetetrahydrofolate reductase gene polymorphisms in children with attention deficit hyperactivity disorder. Int J Med Sci. 2011;8(7):523-528.

9. Young SN. Folate and depression-a neglected problem. J Psychiatr Neurosci. 2007;32(2):80-82.

10. Papakostas GI, Shelton RC, Zajecka JM, et al. L-methylfolate as adjunctive therapy for SSRI-resistant major depression: results of two randomized, double-blind, parallel-sequential trials. Am J Psychiatry. 2012;169(12):1267-1274.

11. Gibson ML, Hohmeier KC, Smith CT. Pharmacogenomics testing in a community pharmacy: patient perceptions and willingness-to-pay. Pharmacogenomics. 2017;18(3):227-233.

12. Yee MM, Josephson C, Hill CE, et al. Cytochrome P450 2D6 polymorphisms and predicted opioid metabolism in African American children with sickle cell disease. J Pediatr Hematol Oncol. 2013;35(7):e301-e305.

13. Gazzaniga D, Brenton A, Meshkin B. A precision medicine approach to a patient with unresolved pain following orthopedic surgery: a case report. J Med Case Rep. 2017;11(1):50. 
The Journal of Pain Research is an international, peer reviewed, open access, online journal that welcomes laboratory and clinical findings in the fields of pain research and the prevention and management of pain. Original research, reviews, symposium reports, hypothesis formation and commentaries are all considered for publication.

Submit your manuscript here: https://www.dovepress.com/journal-of-pain-research-journal

The manuscript management system is completely online and includes a very quick and fair peer-review system, which is all easy to use. Visit http://www.dovepress.com/testimonials.php to read real quotes from published authors. 